\title{
Who is the winner, pre-, post-, or mixed-dilution hemodiafiltration?
}

\author{
Hayne Cho Park, Young-Ki Lee \\ Department of Internal Medicine, Hallym University College of Medicine, Seoul, Republic of Korea
}

\section{See Article on Page 445-456}

Online hemodiafiltration (HDF) is the most technologically advanced renal replacement therapy, combining convective and diffusive solute removal. Online HDF removes a larger volume of small and middle molecule uremic toxins compared to conventional hemodialysis (HD). Recent clinical trials demonstrated that HDF improves patient survival when adequate convection volumes are achieved $[1,2]$. Online HDF is more complex than conventional HD. Various modes of online HDF, differing by the site of replacement fluid infusion, are in use. The replacement fluid can be infused into the tubing downstream of the dialyzer (postdilution), upstream of the dialyzer (predilution), both upstream and downstream (mixed dilution), or into the middle of the dialyzer blood pathway (mid-dilution). Each has its strengths and limitations (Table 1 ).

Postdilution mode is the most common method of fluid substitution in online HDF. Postdilution HDF is the most effective method in terms of solute removal. However, it can increase the viscosity of the blood before fluid substitu- tion, which results in deposition of plasma proteins on the membrane surface, clogging of membrane pores, increased transmembrane pressure (TMP), and occlusion of dialyzer blood channels [1]. Hemoconcentration generally limits the filtration fraction to $20 \%-25 \%$ of the blood flow rate in postdilution HDF [3]. However, modern HDF machines have the option to run TMP-controlled mode during HDF by adapting the substitution flow according to the blood viscosity in the dialyzer [4]. Filtration fraction up to $30 \%$ can be achieved with TMP-controlled mode without excessive hemoconcentration. However, the probabilities of clotting and protein deposition are increased when blood flow is interrupted. For that reason, a high blood flow rate (typically $\geq 350 \mathrm{~mL} / \mathrm{min}$ ) and a well-functioning vascular excess (arteriovenous fistula blood flow of $\geq 600 \mathrm{~mL} / \mathrm{min}$ ) are prerequisites for successful high-volume postdilution HDF [5].

On the other hand, the hemoconcentration associated with postdilution HDF can be avoided by infusing the replacement fluid upstream of the dialyzer in predilution HDF [6]. This reduces the risks of clotting and protein deposition and allows much higher ultrafiltration rates up to $100 \%$ of the blood flow rate [3]. Despite achieving higher

Received: August 10, 2021; Accepted: August 15, 2021

Editor: Tae-Hyun Yoo, Yonsei University, Seoul, Republic of Korea

Correspondence: Young-Ki Lee

Department of Internal Medicine, Hallym University College of Medicine, 1 Singil-ro, Yeongdeungpo-gu, Seoul 07441, Republic of Korea.

E-mail:km2071@naver.com

ORCID: https://orcid.org/0000-0003-3464-6144

Copyright (C) 2021 by The Korean Society of Nephrology

(a) This is an Open Access article distributed under the terms of the Creative Commons Attribution Non-Commercial and No Derivatives License (http:// creativecommons.org/licenses/by-nc-nd/4.0/) which permits unrestricted non-commercial use, distribution of the material without any modifications, and reproduction in any medium, provided the original works properly cited. 
Table 1. Strengths and limitations of online HDF modes

\begin{tabular}{|c|c|c|c|}
\hline Mode of HDF & Postdilution & Predilution & Mixed dilution \\
\hline Advantages & $\begin{array}{l}\text { - Effective in solute clearance \& removal } \\
\text { - Decrease consumption of replacement } \\
\text { solution }\end{array}$ & $\begin{array}{l}\text { - Decrease hematocrit and TMP } \\
\text { - Reduce the risks of clot formation and } \\
\text { protein deposition in dialyzer } \\
\text { - Is available in relatively low blood flow rate } \\
\text { - Reduce membrane stress }\end{array}$ & $\begin{array}{l}\text { - Avoid shortcomings of both predi- } \\
\text { lution \& postdilution modes }\end{array}$ \\
\hline Disadvantages & $\begin{array}{l}\text { - Increase hematocrit and TMP } \\
\text { - Increase the risks of clot formation and } \\
\text { protein deposition in dialyzer } \\
\text { - Require relatively high blood flow rate } \\
\text { - Increase membrane stress (potential } \\
\text { albumin leakage) }\end{array}$ & $\begin{array}{l}\text { - Reduce solute clearance and removal } \\
\text { - Increase consumption of replacement } \\
\text { solution }\end{array}$ & $\begin{array}{l}\text { - Require specific HDF machine } \\
\text { with two infusion pumps } \\
\text { - Require specific blood tubing set }\end{array}$ \\
\hline
\end{tabular}

HDF, hemodiafiltration; TMP, transmembrane pressure.

ultrafiltration rates, predilution HDF reduces the efficiency of both diffusive and convective solute removal because predialysis solute concentrations are decreased or diluted by upstream infusion of substitution volume. For equivalent clearance with the postdilution method, the ultrafiltration rate needs to be increased in predilution HDF at least two times greater than that of postdilution HDF [3].

In mixed dilution HDF, replacement fluid is substituted both upstream and downstream of the dialyzer. This combines the beneficial effects of both predilution and postdilution modes to optimize solute removal. The TMP-controlled mode automatically adjusts and controls the infusion ratio between predilution and postdilution as well as the total infusion volume [7]. Mixed dilution HDF can maximize the total infusion volume while reducing blood hyperviscosity [7]. Mixed dilution HDF results in higher convective removal of small and middle molecule uremic toxins than predilution HDF while maintaining the optimal pressure conditions within the dialyzer [5]. Therefore, mixed dilution HDF can be a good alternative to compensate for the drawbacks of predilution or postdilution HDF modes [6]. Despite these advantages of mixed dilution HDF, few studies have compared the efficacy of mixed dilution HDF versus other HDF modes.

In this issue of Kidney Research and Clinical Practice, Park et al. [8] conducted a randomized controlled trial to compare convection volume and solute clearance between predilution HDF and mixed dilution HDF in patients receiving maintenance HD. The mixed dilution mode was not inferior to the predilution mode considering effective convection volume $(51.5 \pm 9.0 \mathrm{~L} /$ session vs. $41.0 \pm 10.3 \mathrm{~L} /$ session, respectively). In addition, mixed dilution HDF showed higher clearance of $\beta 2$-microglobulin compared to predilution
HDF. The solute removal rate correlates well with convection volume when performing HDF. Although the absolute convection volume was greater in predilution HDF, the effective convection volume from mixed dilution HDF was approximately $20 \%$ higher than that of predilution HDF. Since predilution HDF uses twice as much replacement fluid as the postdilution mode, the effective convection volume for mixed dilution HDF can be calculated as follows as the authors suggested: effective convection volume $=$ substitution volume in predilution mode $+(2 \times$ substitution volume in postdilution mode) + ultrafiltration volume [8] In this regard, the effective convection volume can be used instead of absolute convection volume when comparing the convection volumes between predilution and mixed dilution HDF.

Previous studies well demonstrated an inverse relationship between the convection volume during HDF and the mortality risk [1,2]. In postdilution HDF, the substitution volume of $\geq 23 \mathrm{~L} /$ session is currently recommended [5]. Recently, a few studies evaluated the optimal dose of substitution volume for predilution HDF for improving patient survival. The study conducted by the Japanese Society for Dialysis Therapy found that predilution HDF was associated with improved survival compared to conventional HD with a trend toward improved cardiovascular survival. Patients treated with high substitution volumes ( $\geq 40 \mathrm{~L} / \mathrm{session}$ ) had improved all-cause and cardiovascular survival compared to those treated with lower substitution volumes ( $<40 \mathrm{~L} /$ session). The optimal substitution volume associated with improved overall survival was estimated to be $50.5 \mathrm{~L}$ per session for predilution HDF [9]. However, the optimal convection volume for mixed dilution HDF remains unknown 
since no studies have examined clinical outcomes according to convection volume.

Meanwhile, HDF can lead to significant albumin loss into the dialysate, especially with a highly permeable membrane and high convection volume. Data on albumin loss during HDF are limited and conflicting. Although the serum albumin levels were not different between the predilution and mixed dilution groups, the exact amount of albumin loss via the dialyzer was not evaluated in this study. However, a previous study by Potier et al. [10] demonstrated a higher level of albumin loss in mixed dilution mode compared to predilution mode. Although the degree of albumin loss can be greater in mixed dilution mode, it was within the safety margin $(<5 \mathrm{~g} /$ session $)$.

High-volume HDF is indicated for patients with end-stage renal disease. The clinical benefits of high-volume HDF, particularly postdilution HDF, include improved patient survival and cardiovascular outcomes, better intradialytic hemodynamic stability, less inflammation-related or dialysis-related complications, improved derangement in calcium-phosphate homeostasis and less vascular calcification, better preservation of residual renal function, and improved quality of life [1]. Theoretically, postdilution HDF is the most efficient mode for solute removal. However, successful postdilution HDF depends on high blood flow rates, reliable vascular access, adequate anticoagulation throughout the procedure, and the absence of any condition that increases blood viscosity (high hematocrit, cryoglobulinemia, and gammopathies) [3]. When the prerequisites for postdilution HDF are unavailable, predilution or mixed dilution HDF may be the more appropriate modes. Therefore, predilution or mixed dilution HDF may be the preferable option for patients in Asian countries, such as Korea or Japan, who have relatively lower blood flow of arteriovenous access compared to Caucasians.

The optimal dialysis strategy for patients with end-stage renal disease may differ by country and may not be driven by the best evidence but by experience and local center performance. More evidence for a survival benefit of high-volume HDF is still needed. Currently, the mode of HDF should be decided based on the characteristics and needs of the individual patient.

\section{Conflicts of interest}

All authors have no conflicts of interest to declare.

\section{ORCID}

Hayne Cho Park, https://orcid.org/0000-0002-1128-3750

Young-Ki Lee, https://orcid.org/0000-0003-3464-6144

\section{References}

1. Schiffl H. Online hemodiafiltration and mortality risk in endstage renal disease patients: a critical appraisal of current evidence. Kidney Res Clin Pract 2019;38:159-68.

2. Wizemann V, Külz M, Techert F, Nederlof B. Efficacy of haemodiafiltration. Nephrol Dial Transplant 2001;16 Suppl 4:27-30.

3. Tattersall JE, Ward RA; EUDIAL group. Online haemodiafiltration: definition, dose quantification and safety revisited. Nephrol Dial Transplant 2013;28:542-50.

4. Kim DH, Lee YK, Park HC, et al. Stepwise achievement of high convection volume in post-dilution hemodiafiltration: a prospective observational study. Semin Dial 2021;34:368-74.

5. Schiffl H. High-volume online haemodiafiltration treatment and outcome of end-stage renal disease patients: more than one mode. Int Urol Nephrol 2020;52:1501-6.

6. Canaud B, Lévesque R, Krieter D, et al. On-line hemodiafiltration as routine treatment of end-stage renal failure: why pre- or mixed dilution mode is necessary in on-line hemodiafiltration today? Blood Purif 2004;22 Suppl 2:40-8.

7. Pedrini LA. On-line hemodiafiltration: technique and efficiency. J Nephrol 2003;16 Suppl 7:S57-63.

8. Park KS, Kang EW, Chang TI, et al. Mixed- versus predilution hemodiafiltration effects on convection volume and small and middle molecule clearance in hemodialysis patients: a prospective randomized controlled trial. Kidney Res Clin Pract 2021 Jul 30 [Epub]. DOI: 10.23876/j.krcp.21.044.

9. Kikuchi K, Hamano T, Wada A, Nakai S, Masakane I. Predilution online hemodiafiltration is associated with improved survival compared with hemodialysis. Kidney Int 2019;95:929-38.

10. Potier J, Le Roy F, Faucon JP, et al. Elevated removal of middle molecules without significant albumin loss with mixed-dilution hemodiafiltration for patients unable to provide sufficient blood flow rates. Blood Purif 2013;36:78-83. 